\title{
Quantum Mechanics, Time and Ontology
}

\author{
Valia Allori \\ Detaptment of Philosophy \\ Northern Illinois University \\ vallori@niu.edu
}

\begin{abstract}
Against what is commonly accepted in many contexts, it has been recently suggested that both deterministic and indeterministic quantum theories are not time-reversal invariant, and thus time is handed in a quantum world. In this paper, I analyze these arguments and evaluate possible reactions to them. In the context of deterministic theories, first I show that this conclusion depends on the controversial assumption that the wave-function is a physically real scalar field in configuration space. Then I argue that answers which restore invariance by assuming the wave-function is a ray in Hilbert space fall short. Instead, I propose that one should deny that the wave-function represents physical systems, along the lines proposed by the so-called primitive ontology approach. Moreover, in the context of indeterministic theories, I argue that time-reversal invariance can be restored suitably redefining its meaning.
\end{abstract}

Keywords: symmetries, time-reversal invariance, wave-function realism, primitive ontology.

\section{Table of Contents}

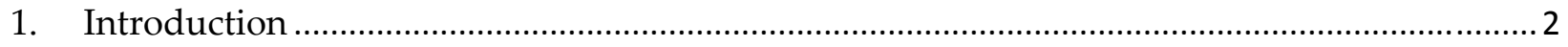

2. The Arguments that Quantum Theory is Time-Reversal Invariant....................................... 3

3. The Arguments that Quantum Theory is not Time-Reversal Invariant................................. 4

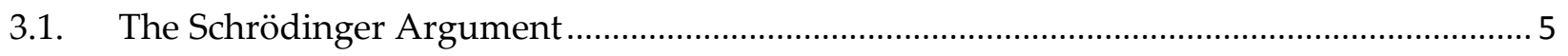

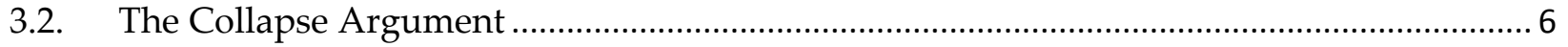

3.3. Generalization to These Arguments to Quantum Theories without Observers .................. 7

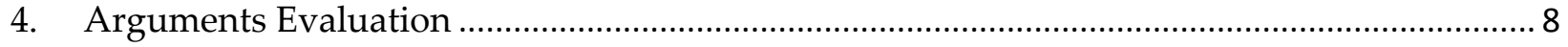

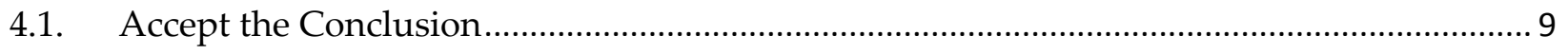

4.2. Reject the Definition of Time-reversal Operator ................................................................. 9

4.3. Reject that the Wave-function is in the State .................................................................... 11 


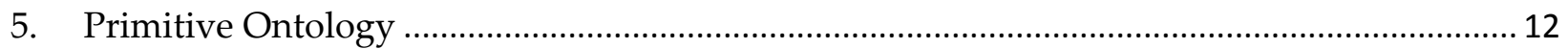

5.1. The Case for Time-Reversal in Deterministic Theories ...................................................... 13

5.2. The Case for Time-Reversal in Indeterministic Theories .................................................. 16

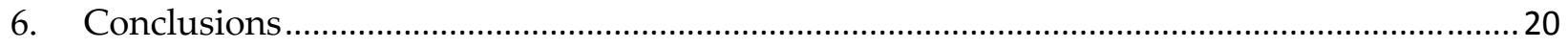

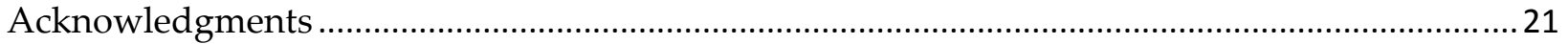

\section{Introduction}

In the context of naturalized metaphysics when discussing the nature of time it is common to consider Einstein's theory of general relativity rather than quantum mechanics. What, if anything, does quantum mechanics tell us about the nature of time? In this paper, I am interested in a particular argument according to which if quantum mechanics is correct, then time has a preferred direction.

In this paper I analyze these arguments in detail and I evaluate possible reactions to them. In doing so, considering deterministic theories, I show that the argument crucially relies on the controversial assumption that the wave-function represents a physical scalar field in configuration space. Time-reversal invariance could be restored by still maintaining that the wave-function is physically real but insisting that it is a ray in Hilbert space rather than a scalar field. In this way it would be natural for the timereversal operator to act on the wave-function as to preserve invariance, namely to complex conjugate it. However, I argue that this strategy has the extremely counterintuitive consequence that there is mismatch between the ontology inferred from the forward history and the one from the backward history of the world. I thus suggest that a more promising move is to deny that the wave-function is physically real to start with, along the lines of a recent approach to the metaphysics of quantum physics based on the notion of primitive ontology. In addition, in the case of indeterministic theories, while the argument against invariance could still stand, I show how one could provide an alternative definition of time-reversal symmetry for indeterministic theories which would restore invariance. If that is right, it follows that quantum theories do not lack time-reversal invariance, and thus it is premature to conclude that time has a preferred direction.

Here is the organization of the paper. In Section 2, I present the arguments that are usually provided for the time-reversal invariance of orthodox quantum mechanics. In Section 3, I articulate the arguments put forward to show that it is not the case, both for orthodox quantum theory and for the various interpretations. Next I discuss various ways to react to these arguments, and then in Section 5 I introduce the one that I take to be more promising, based on the primitive ontology approach. In that section I first 
discuss deterministic theories and then indeterministic ones, showing how there is a sense in which they are both time-reversal invariant. I conclude the paper with a summary of what has been accomplished.

\section{The Arguments that Quantum Theory is Time-Reversal Invariant}

Quantum mechanics as formalized by von Neumann (1932) is known among philosophers of physics as 'orthodox quantum mechanics' (OQM). According to OQM, every physical object is described by a mathematical entity called the wave-function $\psi$, which evolves in time according to two different evolution equations: when the system is undisturbed, the wave-function evolves according to the deterministic Schrödinger equation; when an experiment is performed the wave-function follows an indeterministic stochastic evolution called 'collapse rule' or 'collapse postulate.'

To see whether the theory is time-reversal invariant, let's start with the definition of time-reversal symmetry. Call $S$ the state of the system, which provides the complete description of the system. This symmetry is (arguably, see later) implemented by an operator, $T$, which flips time in $S$ so that $T(S(T))=S(-t)$. Roughly, an equation is timereversal invariant if it treats the past and future as mirror images of each other. A history of the world provided by the theory is composed of a sequence of states ordered in time from the initial time $i$ to the final time $f: S_{i}, \ldots, S_{f}$. A theory is time-reversal invariant just in case given a sequence of states that constitutes a solution for the system, $S_{i}, \ldots, S_{f}$ the sequence of temporally reflected states $T\left(S_{f}\right), \ldots, T\left(S_{i}\right)$ is also a solution. This is commonly explained using a movie analogy: if $S_{i}, \ldots, S_{f}$ is a movie of the world projected forward in time, then the movie projected backward $T\left(S_{f}\right), \ldots, T\left(S_{i}\right)$ is also a possible history of the world. That is, an observer would not be able to rule out the latter as an empirically inadequate characterization of the world. To provide an example, consider classical mechanics, where the state is $S_{t}=(x(t), v(t))$. Instantaneous positions will not change sign with $T$ but velocities, being the rate of change of positions in time, will: $T(S)=$ $T(x(t), v(t))=(x(t),-v(t))$. Thus, if someone films the trajectory of ball 1 hitting ball 2 and they project it backwards ${ }^{1}$, they will see ball 2 hitting ball 1 . As far as one can tell, this is what could have actually happened. That is, the original movie and the time-reversed movie depict two possible ways the world could be. Thus, the theory (in this case classical mechanics) is time-reversal invariant.

Turning now to OQM, the wave-function, since it provides the complete description of any physical system, defines the state $S$. Both the Schrödinger equation and the collapse postulate describe what the wave-function will do under certain

\footnotetext{
${ }^{1}$ Avoiding the initial time in which ball 1 is initially hit by the pole and then the final time in which the balls slow down due to friction.
} 
circumstances, so let us analyze them one at a time. First, if we just switch $t$ to $-t$ (and do nothing else), an overall minus sign shows up in the Schrödinger equation. Because of this, the solutions of the time-reversed Schrödinger equation are not solutions of the original one. In order to get solutions we also have to replace the wave-function with its complex-conjugate $\psi^{*}: T(\psi(t))=\psi^{*}(-t)$. The (traditional) response to this is to revise the action of $T$ on the state, and claim that time-reversal also transforms the wave-function into its complex-conjugate. This transformation is implemented by an anti-unitary operator such that $T(\psi, \phi)=(\psi, \phi)^{*}$ (Wigner, 1959). In this way, if $\psi(t)$ is a solution of the Schrödinger equation, also $\psi^{*}(-t)$ is one, and OQM is time-reversal invariant. Following this lead, Earman (2002) and Roberts (2017) have argued that there are two reasons why $T$ acts in this way: a mathematical reason, as well as a physical reason. The former is that wave-function is a ray in Hilbert space, that is an equivalent class of vectors related by a phase $\theta$ of the form $\psi=\left\{\psi e^{i \theta}\right\}$, and because of this one can prove Wigner's theorem as well as Uhlhorn's theorem, from which one can derive that $T$ is anti-unitary (see Roberts 2017 for details). The physical reason is that the information about the temporal direction is contained in the phase of the wave-function, and therefore the phase much change sign when time is reversed (Earman 2002).

Now consider the collapse postulate. By definition, it is not symmetric in time: it specifies the probability of getting a given experimental result after a measurement, not before. The theory predicts, it does not retrodicts: it specifies what the system will look like in the future, given the past (forward probabilities), not what it had looked in the past, given the future (backward probabilities). Nonetheless, one can suitably introduce backward probabilities as follows, in the so-called 'time-symmetric' OQM (Aharonov, Bergmann and Lebowitz, 1964): take two times, $t_{1}$ and $t_{2}$, and a time $t_{3}$ in between; add forward probabilities and backward ones as the probabilities for transitioning to $t_{3}$. In this way one finds a new probability formula, called the ABL formula, for an outcome at a given time conditioned on the earlier state at $t_{1}$ and the later state at $t_{2}$, restoring in this way time-reversal invariance of OQM.

\section{The Arguments that Quantum Theory is not Time-Reversal Invariant}

It has been argued (Callender 2000) that both (sets of) arguments for time-reversal invariance fail. If so, there is a preferred direction of time built in directly into the fundamental laws of the theory, and thus time is handed. In the following I reconstruct his arguments both for deterministic and indeterministic theories. Even if this argument (and the one in the next section) is well-known, I think it one needs to be explicit about each assumption made, in order to analyze the plausibility of the various possible reactions discussed in the next section. 


\subsection{The Schrödinger Argument}

In the previous section we have seen how Wigner's definition of time-reversal could make OQM time-reversal invariant by requiring that the wave-function would transform into its complex-conjugate under $T$. Nevertheless, Callender's argument goes, why should the wave-function transform like that? As we have seen in the previous section, this seems connected to the fact that the wave-function is a ray in Hilbert space. However, Callender continues, the wave-function is a function of the coordinates of all particles, that is $\psi=\psi\left(r_{1}, \ldots, r_{N}\right)$. As such, it is naturally understood as a scalar field in configuration space. This is the position commonly called wave-function realism. ${ }^{2}$ If so, it is not the right kind of object to transform into its complex-conjugate under $T$ : a scalar field should not change its amplitude at all under $T$. What $T$ should do is only switch $t$ into $-t$ when acting on the state $S$, and nothing more. Since in order to have time-reversal invariance the wave-function would have to change under $T$, the argument concludes, then the Schrödinger equation is not time-reversal invariant. In short, here is my reconstruction of the argument that $\mathrm{OQM}$ is not time-reversal invariant (in square brackets there is a sketchy justification for each step):

\section{The Schrödinger Argument:}

1. The complete description of any system is given by its state $S$ [definition of state]

2. What is in the state $S$ represents physical objects [ontological assumption]

3. In OQM the wave-function $\psi$ provides the state $S$ [the two fundamental equations of OQM are equations for the evolution of the wave-function]

4. When no experiments are performed, the wave-function evolves according to the Schrödinger equation [definition of the theory]

5. The time-reversal transformation $T$ transforms a sequence of states into their temporally reversed sequence: $S_{i}, \ldots, S_{f} \rightarrow T\left(S_{f}\right), \ldots, T\left(S_{i}\right)$ [definition of $T$ on a sequence of states]

6. $T$ changes $t$ in the state into $-t: T(S(t))=S(-t)$ [definition of $T$ on a single state: the ontology does not change depending on whether we look at the forward or backward sequence]

7. A deterministic theory is time-reversal invariant if forward solutions are mapped into backward solutions [definition of time-reversal invariance]

8. This does not happen unless we change $\psi$ with its complex conjugate $\psi^{*}$ [mathematics]

9. The wave-function $\psi$ is a scalar field [it is a function on configuration space]

10. If 9 , then there is no reason why $\psi$ should transform into its complex conjugate $\psi^{*}$ under $T$ [physical meaning of scalar field]

2 See Albert (1996, 2013, 2015); Lewis (2004, 2005, 2006, 2013); Ney (2012, 2013, 2015, 2017); North (2013). 
11. Therefore, when OQM describes system that are not observed, it is not time-reversal invariant.

\subsection{The Collapse Argument}

Callender's argument continues by pointing out that the ABL formalism is not really a solution of the problem of backwards probabilities, given that it is just a reformulation of the usual formalism of OQM and as such is not able to tell us which, whether the forward formula or the backward formula, is a fundamental law. If so, given that the collapse postulate is a fundamental law of OQM, it follows that OQM is not time-reversal invariant.

In addition, one should notice that the meaning of time-reversal invariance of an indeterministic theory is not obvious, since the definition outlined above for deterministic theory would not be directly applicable to indeterministic theories. In fact, Callender argues, an indeterministic equation describes a situation in which the past is fixed and the future open. Unlike deterministic theories, in which we have one past and one future which are 'treated the same' by the theory, in indeterministic theories there is just one past but there are multiple futures open to us, each of them with a different probability. The past $S_{i}$ evolves into $S_{f}$, one of the possible futures, with probability $p_{f}$ strictly less than 1 , but once we have reached $S_{f}$, the past $S_{i}$ is determined with probability identical to 1. In the words of Callender:" [...] in general there is no way of evolving from the collapsed system back to the uncollapsed system with the same chance. According to collapse theories, there is a preferred orientation to time." More formally, in Callender's view, an indeterministic theory is time-reversal invariant just in case the probability of a possible sequence of states $S_{i}, \ldots, S_{f}$ is the same as the probability of the temporally reflected sequence $T\left(S_{f}\right), \ldots, T\left(S_{i}\right): \operatorname{prob}\left(S_{i}, \ldots, S_{f}\right)=\operatorname{prob}\left(T\left(S_{f}\right), \ldots, T\left(S_{i}\right)\right)$. This definition reduces to the definition for determinist equations, given that the probabilities would add up to 1 . Given that these probabilities are not the same in OQM, the theory is not time-reversal invariant. Thus, we have, schematically:

The Collapse Argument:

1. The complete description of any system is given by its state $S$ [definition of state]

2. What is in the state $S$ represents physical objects [ontological assumption]

3. In OQM the wave-function $\psi$ provides the state $S$ [the two fundamental equations of OQM are equations for the evolution of the wave-function]

4. When an experiment is performed, the wave-function evolves according to the collapse rule, which is indeterministic [definition of the theory] 
5. The time-reversal transformation $T$ transforms a sequence of states into their temporally reversed sequence: $S_{i}, \ldots, S_{f} \rightarrow T\left(S_{f}\right), \ldots, T\left(S_{i}\right)$ [definition of $T$ on a sequence of states]

6. $T$ changes $t$ in the state into $-t: T(S(t))=S(-t)$ [definition of $T$ on a single state: the ontology does not change depending on whether we look at the forward or backward sequence]

7. An indeterministic theory is time-reversal invariant just in case the probability of a possible sequence of states $S_{i}, \ldots, S_{f}$ is the same as the probability of the temporally reflected sequence $T\left(S_{f}\right), \ldots, T\left(S_{i}\right)$ [definition of time-reversal invariance for indeterministic theories]

8. The collapse postulates generates forward probabilities and not backwards one [definition of collapse postulate];

9. Therefore, when OQM describes system that are observed, it is not time-reversal invariant.

To sum up, if the arguments in sections 3.1 and 3.2 are correct then OQM is not timereversal invariant: both because of the collapse postulate but also, and perhaps more surprisingly, because of the Schrödinger equation.

If OQM is the fundamental theory of the world, then one could also make the further step of inferring that the time has a preferred direction: this is what the quantum revolution has to say about time! However, many consider OQM as very unsatisfactory, ultimately because it is fundamentally vague about when the collapse postulate is supposed to be applied. Allegedly, the collapse happens when an observation is made, but isn't an observation another piece of reality that should be described by the theory, rather than be part of its postulates? Luckily, there are other quantum theories that, unlike OQM, do not need the collapse postulate and any reference to the observer or the act of observation. Because of this, they are sometimes called 'quantum theories without observers' (Popper 1967). Thus, instead of drawing metaphysical conclusion from a dubious theory, let us focus on these more precise, observer-free quantum theories, namely the pilot-wave theory, the many-worlds theory and the spontaneous collapse theory.

\subsection{Generalization to These Arguments to Quantum Theories without Observers}

In the pilot-wave theory ${ }^{3}$ the description provided by the wave-function is supplemented by the position of particles. Accordingly, the temporal evolution of a given physical system is governed by two fundamental deterministic equations: the wave-function

\footnotetext{
${ }^{3}$ The theory was originally proposed by de Broglie (1928), then developed by Bohm (1952). Recently, it has been defended by Valentini (1991) and by Dürr, Goldstein and Zanghí (1992).
} 
evolves according to Schrödinger's equation, while the particle positions evolve according to the so-called guidance equation, which determines the particle velocity in terms of the wave-function. Unlike OQM, the collapse postulate is not fundamental in this theory, but it follows from the fundamental laws of motion. If so, therefore, there is no problem of backward probabilities. However, as in OQM, the Schrödinger equation is fundamental in this theory, and one could run an argument similar to the Schrödinger argument of Section 3.1 just replacing in premises 3 and 4 'orthodox quantum mechanics' with 'pilot-wave theory'.

Another well-developed deterministic quantum theory without observer is the many-worlds theory. ${ }^{4}$ In this theory the law of evolution for the wave-function is given by the Schrödinger equation alone. Roughly put, the macroscopic superpositions created by the linearity of the Schrödinger equation do not create a problem because they realize in different 'worlds' which do not interact with one another. Thus, there is no fundamental collapse of the wave-function; however, we still have the Schrödinger evolution. Because of this, assuming that the wave-function describes the state of the system, and is a scalar field, one can run a very similar argument as the one in Section 3.1 and conclude that the theory is not time-reversal invariant.

Another quantum theory that does not invoke the observer in its formulation is the spontaneous collapse theory, also called spontaneous localization theory or GRW theory, from the names of Ghirardi, Rimini and Weber who first proposed it. ${ }^{5}$ In this theory, the wave-function evolves according to the Schrödinger equation until a random time at which the wave-function undergoes an instantaneous collapse: it localizes around a random point. The rate of collapse depends on how big the object under consideration is, and because of that, the collapse postulate is not fundamental. Nonetheless, according to Callender (2000), the theory is not time-reversal invariant, as one can see applying the collapse argument in Section 3.2 suitably modifying premises 3, 4 and 8. In fact, like any other indeterministic theory, it describes a situation in which the past is fixed and the future open.

\section{Argument Evaluation}

Now that we have the arguments spelled out in premise-conclusion form, it will be easier to systematically assess them. In this section I focus on the Schrödinger argument, leaving the responses specific to the indeterministic case to Section 5.2. Some initial, but unpromising, reactions from those who would want to block the conclusion could be the following. One could question that the state is connected to the ontology (premise 1 or 2

\footnotetext{
${ }^{4}$ This theory was first proposed by Everett (1957), and most recently has been defended most notably by deWitt (1970), Lockwood (1989), Saunders (1993), Deutsch (1996), and Wallace (2012).

${ }^{5}$ Ghirardi, Rimini and Weber (1986). See also Pearle (1989).
} 
of any of the arguments in sections 3.1 and 3.2). Nevertheless, this reply is difficult to defend, since scientific realism is assumed in these arguments. In principle, one could also reject that the definition of time-reversal transformation is correct (premises 5). However, there seems to be little evidence that this might be the case. ${ }^{6}$ In addition, one could reject the definition of time-reversal invariance of a theory (premise 7). While there is little support for this move in the context of deterministic theories, I argue in Section 5.2 that this is what needs to be done in indeterministic theories to restore invariance.

\subsection{Accept the Conclusion}

Another possible reaction to these arguments is simply to accept them. Arguably, people have been suspicious about them because symmetries are seen as being somewhat untouchable. However, one could question the necessity or the desirability of symmetries: why do we want a theory to have a given symmetry? Indeed, why should we want symmetries at all? Isn't just an arbitrary stipulation that theories should have invariance properties? One could argue that if the most natural reading of the elements in the theory, namely identifying the wave-function as a scalar field, shows us that the theory lacks symmetries, so be it.

However, I believe that this view is difficult to defend. In fact symmetries have always had an important role in theory construction: think of the growing use of symmetry groups in the development of new theories. ${ }^{7}$ Also, symmetries are used in theory evaluation as well: the history of science is full of examples of otherwise successful theories that have been rejected because they were not invariant under some important transformation. ${ }^{8}$ Thus, all other things being equal, rejecting symmetries seems to be an unnecessarily radical move.

\subsection{Reject the Definition of the Action of the Time-reversal Operator on the State}

More promising, I think, is to reject premise 6, namely that $T$ merely flips the sign of $t$ in the state. This is indeed the route taken by Wigner and the supporters of the traditional view of time-reversal invariance in OQM such as Earman and Roberts (see also Aharonov and Rorhlich 2005) already mentioned in Section 2.

\footnotetext{
${ }^{6}$ Nonetheless, it should be noted that North (2008) argues, even if in a different context, that the source of disagreement between Albert (2000) and Malament (2004) about the time-reversibility of classical electrodynamics is indeed that they endorse two different notion of time-reversal transformation. However, see Allori (2015b) for a discussion.

${ }^{7}$ However, for a dissenting view, see Hossenfelder (2018).

${ }^{8}$ For a general discussion of the importance of symmetries in scientific reasoning, see Brading and Castellani (2005).
} 
This position has been challenged, as we saw in Section 3, and Roberts (2017) has defended it by insisting that the time-reversal operator acts on the state as the nature of the state dictates (see also Skow 2010 in the context of Galilean symmetry) but disagreeing with Callender as to which object the wave-function is: not a field on config ration space but a ray in Hilbert space. If so, then it is natural for $T$ to act on it as to make the theory time-reversal invariant: $T(\psi(t))=\psi^{*}(-t)$.

The argument at this point could go both ways, however it should be noted is that the premise-conclusion forms of the arguments against invariance make apparent a problem for Roberts' view. In fact, consider the total history of the world, which is given by a sequence of instantaneous states. Here, we are assuming that the wave-function belongs to the state and therefore each state will tell you what the word is at an instance, as described by the wave-function at that time. In this regard, it seems reasonable to assume that a state taken from the backward sequence at one time, and the state taken from the forward sequence at the same time would provide exactly the same picture of the world: why would the image of the world change, depending on whether I look at it from the forward or the backward movie? However, look at the backward history. As we have just seen, being the wave-function a ray in Hilbert space, it transforms under $T$ into its complex conjugate. This is what does the trick to maintain time-reversal invariance. So, a backward sequence will be one in which each state at a given time is time-reversed and complex-conjugated. That means that the state from the backward sequence will be different from the one from the forward sequence. In other words, if one accepts the definition of time-reversal which allows $T$ to also complex-conjugate the wave-function, then one also has to accept that $T$ is able to change the ontology, given that the state of a system represents its ontology and that $S$ as taken from the forward sequence is different from $S$ as taken from the backward one. This is extremely strange, since it amounts to saying that the content of a state of the world could change depending on whether it comes from the forward or the backward movie of the world. This is at best counterintuitive: how is it possible that the wave-function in the state of the forward movie has one value, but in the states of the backward one it has another value? A very similar debate is taking place in the context of classical electrodynamics. ${ }^{9}$ There is in fact an argument proposed by Albert (2000) to the conclusion that classical electrodynamics is not time-reversal invariant, which is very similar to the Schrödinger argument of Section 3.1 when one substitutes the magnetic field with the wave-function. It can be briefly summarized as follows: if the magnetic field $B$ is physically real, and it is represented by a vector function, then there is no reason for it to flip sign under $T$ as assumed by physics books; therefore $B$ should not flip sing under $T$ and, Albert concludes, classical electrodynamics is not time-reversal invariant. In this context Earman (2002), Arntzenius (2004) and Malament (2004) have not disputed the physical reality of $B$

${ }_{9}^{9}$ See Allori (2015b) for a discussion. 
but its identification with a vector field: it is rather a pseudo-vector, and this is the reason of its 'peculiar' transformation under $T$. However, this implies that if one takesa state from the forward sequence of the world and reads off from it the ontology, she would obtain a different result than if she considered a state from the backward sequence. In fact, from the forward sequence she would find " $B$ ", while from the backward one she would find " $-B$. ." But why would it be?

Moreover, both in classical electrodynamics and in quantum mechanics, the question remains of what kind of physical entity can be interpreted as being represented by a pseudo-vector, in the case of the magnetic field, or by a ray, as in case of the wavefunction. In other words, it is straightforward that a point in three-dimensional space mathematically may represent some material entity, such as a point-particle: a particle takes just one parameter to be described. Moreover, it is also straightforward that a scalar function may represent, say, a matter density field, because such a field associates to each point in space a scalar number. Similarly, a vector function may represent, say, an electric field. However, can an equivalent class of functions that differ by some constant represent something physical? The answer seems to me to be negative, at least if one look at potentials. They are defined in this way, but they are not taken to represent some physical entity; rather they are part of the law of motion. The situation seems similar here: what is a pseudo-vector able to define? What about a ray? I think the best answer to this is that they are not suitable to represent anything material, like potentials in classical theories. ${ }^{10}$

\subsection{Reject that the Wave-function is in the State}

This leads us directly to another possibility: one could deny premise 3 and maintain that the wave-function is not in the state, namely that the wave-function is not physically real. This is one crucial assumption of Callender's argument which, prima facie, seems indisputable: isn't the wave-function what quantum theories are about, after all? However, a recent approach to the metaphysics of quantum mechanics proposes just that: the wave-function does not represent physical entities. I am referring to the so-called primitive ontology approach, according to which matter in a quantum theory is represented by a spatio-temporal entity, the primitive ontology of the theory, never by the wave-function. ${ }^{11}$ It is controversial what the wave-function is, but it never represents matter. ${ }^{12}$ Be that as it may, let's discuss in the next section how the primitive ontology

\footnotetext{
${ }^{10}$ See Allori (2018) for an elaboration of a similar point in the context of Galilei invariance of quantum mechanics.

${ }^{11}$ See Dürr, Goldstein, and Zanghí (1992); Allori, Goldstein, Tumulka and Zanghí (2008); Allori (2013a), (2013b), (2015a).

12 Some claim it has a nomological role (Dürr, Goldstein and Zanghí 1997, Goldstein and Teufel 2001, Goldstein and Zanghí 2013). See also Miller (2014), Callender (2015), Bohgal and Perry (2017) for a
} 
approach can provide a rebuttal of the arguments against the time-reversal invariance of quantum mechanics.

\section{Primitive Ontology}

In this section I present how the primitive ontology approach works in the various deterministic theories without observers, starting with the pilot-wave theory. In this framework, the pilot-wave theory is seen as a theory of particles, which constitute the primitive ontology, while the wave-function enters the theory in the definition of the guidance equation which determines the motion of the particles. In this picture, tables and chairs are not made of wave-functions but of particles, mathematically represented in the theory by their positions in three-dimensional space.

The proponents of this approach think that the other quantum theories without observers should be framed as theories about the behavior of some spatiotemporal threedimensional entity (the primitive ontology) and not about the wave-function, which 'only' serves the function of defining the evolution equation for the primitive ontology. This is a radical departure from how these theories are usually regarded, given that both many-worlds and the spontaneous localization theory have equations governing the evolution of the wavefunction and nothing else, and have thus interpreted as theories about the wave-function. In contrast, this approach has been taken to imply that one needs always to add something to the wave-function, and because of this it has been criticized. ${ }^{13}$ However, since the wave-function does not represent matter in this approach, it's not that one adds something to the wave-function but rather that one adds a suitable ontology to a theory that previously had none. Be that as it may, I will not discuss in this paper the motivations for the view or how one can defend it, since this has been done elsewhere. ${ }^{14}$ What is of importance here is that one consequence of this view concerns the symmetry properties of a theory, and in particular the time-reversal symmetry. Before discussing this connection in the next sections, let me elaborate a bit more on how manyworlds and the spontaneous localization theory look like in this framework.

In this approach, the many-worlds theory may have different ontologies: particles, matter fields, or spatiotemporal events (dubbed 'flashes'), each with their own evolution equation, implemented by a Schrödinger-evolving wave-function. Each different ontology selects different many-world theories, so one has a particle-many-worlds theory, a matter density-many-worlds theory, or a flash-many-worlds theory. It is still sensible to label all of these theories as 'many-worlds' because there is a sense in which

\footnotetext{
Humean perspective, and one according to which the wave-function is a property (Monton 2013, Lewis 2013, Esfeld, Lazarovici, Hubert Dürr 2014, Suàrez 2015).

${ }^{13}$ See Lewis (2017) and references therein.

${ }^{14}$ See, e.g. Allori (2103a), (2013b), (2015a).
} 
they describe different realities in the same spacetime. For instance, one could construct a theory, called Sip, in which matter is made of particles (hence the ' $\mathrm{p}$ ' in the name) whose temporal evolution is guided by a Schrödinger evolving wave-function (hence the ' $S$ '). However, in contrast with the pilot-wave theory, here there is no trajectory connecting one particle at one time with that particle at another time. Rather, at each time one is given a distribution of particle configuration, and the distributions at different times are uncorrelated or independent (hence the ' $i$ '). ${ }^{15}$ This theory presents a many-world's character. In fact, due to the linearity of the Schrödinger evolution, the wave-function may be in superpositions of components very far apart in configuration space connected to unrealized states of affairs. Since in Sip there is no connection whatsoever between what there is at a given instant of time and what there is at the previous or following instant, there is a very high probability that particle will visit in every second those distant regions. Therefore, at time $t$ there can be cities and people and at time $t+d t$ they have disappeared. So, many worlds exist one after another. Another possible many-world theory is $\mathrm{Sm}$, in which a Schrödinger evolving wave-function governs the behavior of a matter field ontology $m$ (defined in terms of the wave-function). ${ }^{16}$ This theory involves many worlds because the superpositions of the wave-function get inherited by the matter field, and they effectively do not interact due to decoherence effects. Because of this, they constitute different worlds, which are, so to speak, reciprocally transparent. A third many-worlds theory is Sf, which involves an ontology directly defined in space-time, called the 'flashes', which are spatio-temporal events 'full of matter.' The distribution of these flashes is provided by a rule defined by a Schrödinger evolving wave-function. ${ }^{17}$ Like in Sm, the superpositions of the wave-function are inherited by the flashes, and by the linearity of the Schrödinger evolution the flashes form independent families of correlated flashes associated with the terms of the superposition, with no interaction between the families. Thus, they can indeed be regarded as comprising many worlds, superimposed on a single space-time.

\subsection{The Case for Time-Reversal in Deterministic Theories}

As we have seen, a key component of this approach is that the wave-function has been demoted from its ontological status: it does not represent matter. This has consequences for the symmetry properties of the theory: symmetries have to do with invariances of

\footnotetext{
${ }^{15}$ This theory was originally proposed by Bell (1987) and later dubbed 'Sip' in Allori Goldstein Tumulka Zanghi (2008). 'S' comes from the fact that the wave-function in this theory evolves according to Schrödinger's equation, ' $\mathrm{p}$ ' from the fact that matter is made of particles, and ' $\mathrm{I}$ ' refers to the fact that their locations are independent from one another.

16 See Allori, Goldstein, Tumulka and Zanghí (2008).

17 See Allori, Goldstein, Tumulka and Zanghí (2008).
} 
possible pictures of the world, and since the wave-function has only an indirect role in giving us these pictures, it should not be our focus when discussing symmetries. In other words, the symmetries of the theory should be determined by its primitive ontology, not by the wave-function. It is thus wrong to focus on the fundamental equation for the wavefunction when looking at symmetry properties; rather, one should focus on the law of evolution of the primitive ontology. To be more precise, to say that a deterministic theory has a given symmetry is to say that the possible histories of the world, those which are allowed by the theory, when transformed according to the symmetry, will again be possible histories. If one takes these histories to be described by the behavior of the wavefunction, ends up to deny time reversal invariance, as we have seen. However, in this framework, the possible histories of the world are given solely by the primitive ontology. The possible histories are the ones that are empirically adequate, and a theory has a given symmetry if empirically adequate histories are mapped into other empirically adequate histories. In this approach, empirical adequacy is obtained via a balance between the choice of the primitive ontology and of the laws (both for the primitive ontology and the other objects in the law such as the wave-function). The primitive ontology is selected as the overall simplest and most explanatory choice among the alternatives.

So, in the case of the pilot-wave theory and of $\mathrm{Sm}$, to check for symmetries one does not look at the Schrödinger equation, because that is the equation for the wavefunction. Rather, one considers the guidance equation and the equation for the matter density respectively. This allows for the variables in the theory other than the primitive variables, in this case the wave-function, to transform under the symmetry however it is necessary so that the histories of the primitive ontology can still be possible histories of the theory. That is, one looks for the transformation of the wave-function which will preserve the symmetry of the theory. As mentioned earlier, in this approach one assumes that symmetries are a desired requirement for the theory, in line with what many physicists believe. In the case of time-reversal transformation $T$, in order for the pilotwave theory - for instance - to be time-reversal invariant, the wave-function needs to transform under $T$ into its complex conjugate: $T(\psi(t))=\psi^{*}(-t)$. In other words, the $T$ transformed solutions of the guide equations will also be solutions of the original guide equation just in case the wave-function transforms that way. If the wave-function were to remain the same, as it seems it should if it is a scalar field, then the theory will lose this symmetry. In this approach, instead, the wave-function does not represent matter, but rather helps represent the law of motion for it. As such, then, there are no special restrictions on how it should or should not transform under $T$ or any other symmetry transformation. The other possibility would be that the wave-function is in the state but it is a ray, preserving the symmetry. However, as we have seen, this leads to the problem of mismatch: a state taken from a forward sequence is not identical to the corresponding state in the backward sequence, thus the ontology changes depending if we see the 'movie' backward or forward. In contrast, here since the wave function is never in the 
state, and matter is represented by the primitive ontology whose description does not change in the backward and the forward story. ${ }^{18}$

Also, it is important to stress how this approach is able to explain why the traditional arguments for time-reversal, like the ones proposed by Earman and Roberts, work, without falling short as they do. In fact, in the context of classical electrodynamics, this approach can account for why $B$ is represented by a pseudo-vector (which is what was required for time-reversal): not because the time-reversal operator acts differently depending on whether the state is taken from the forward or the backward movie, but because $B$ is not in the state and if it weren't a pseudo-vector, then the theory would not be time-reversal invariant. Similarly, in the quantum domain one makes sense of the reason why, as assumed by Earman, Roberts, Aharonov and Rorhlich and by the majority of physicists, the wave-function is a ray in Hilbert space, namely because it is not in the state and it needs to transform as a ray would in order to preserve the relevant symmetry. In other words, the primitive ontology approach explains that the reason why $B$ and $\psi$ are the mathematical objects they are, namely a pseudo-vector and a ray, respectively. Indeed, it is because these objects inherently transform in such a way as to recover empirically adequate trajectories also preserving their symmetry properties. In particular: in classical electrodynamics the magnetic field transforms in such a way that the theory is time-reversal invariant; and in deterministic quantum theories, such as the pilot-wave theory and Sm, the wave-function transforms so that these theories possess the time-reversal symmetry. In other words, we can come back to the question asked at the end of Section 4.2. Thus, instead of asking: "Can a ray mathematically represent physical object?" we can ask: "What kind of entity is represent by a ray in Hilbert space?" As we have anticipated, the most natural answer comes from the observation that other quantities, such as potentials, are defined as equivalence classes and taken to be part of the law of motion rather than representing matter. So presumably one could take a similar stand here and argue that it is not surprising that the wave-function is mathematically a ray in Hilbert space only if one takes the wave-function not as representing physical objects. Indeed, it is because it doesn't represents physical objects that it makes sense it is

\footnotetext{
${ }^{18}$ Notice that one could take the primitive ontology approach to maintain that the wave-function is in the state (premise 3 is true) but that only part of the state, but not all, is physically real (denying premise 2). Specifically, the wave-function, even if it belongs to the state, does not represent physical objects. However in this case one also would need to change the definition of $T$ and how it acts (premises 5 and 6) so that $T$ does not act on the state but only on the ontology $O$. I this way one would have:

$5^{\prime}$ ) The time-reversal transformation $T$ transforms a sequence of ontologies $O$ into their temporally reversed sequence: $O_{i}, \ldots, O_{f} \rightarrow T\left(O_{f}\right), \ldots, T\left(O_{i}\right)$ [definition of time-reversal transformation $T$ on a sequence of ontologies]

$\left.6^{\prime}\right) T$ changes $t$ in the ontology into $-t: T(O(t))=O(-t)$ [action of $T$ on a single ontology: the ontology does not change depending on whether we look at the forward or backward sequence].
} 
a ray: it is the right kind of object that produces empirically adequate trajectories for the primitive ontology and preserves time-reversal invariance symmetry.

This discussion may also answer an objection that one may have, namely that this account seems to be circular: one defines the action of the time-reversal operator on the wave-function such that the theory is invariant, while the real question is to justify why the operator acts the way it does on it. ${ }^{19}$ That is, one may insist that an operator such as $T$ should act on an object as the nature of the object prescribes (Skow 2010), and therefore one should explain which kind of object the wave-function should be in order to transform as to preserve invariance. As pointed out above, the answer is: the wavefunction is not a scalar field, rather it is a ray in Hilbert space. And, in contrast to what is proposed by the rival approach by Earman and Roberts, this view explains why it is a ray, namely because of its role is to generate the evolution of the primitive ontology and it does that so that the theory is invariant. Notice that the account would be circular if the wave-function were in the state, and thus we would need to define what the wavefunction is beforehand. However, in this approach, the wave-function enters at a later stage to implement the motion of the ontology, but it is not part of the ontology in itself. Therefore the theory is invariant because, by denying that the wave-function belongs to the state, the states in the sequence which constitutes the history of the world includes merely the primitive ontology, and whatever action $T$ has of the state does not involve the wave-function.

\subsection{The Case for Time-Reversal in Indeterministic Theories}

Be that as it may, the story is different with Sip and Sf which instead are indeterministic theories (in the former the configuration jumps at random, and in the latter the distribution of the flashes is probabilistic). Let's see how to deal with indeterministic theories through the example of the spontaneous collapse theory as discussed within the primitive ontology framework. What is matter made of in this theory, if not the wavefunction? As in the case of the many-worlds theory, different proposals have been put forward. One, called GRWm, is a theory in which physical objects are described by a matter density distribution $m$ in three-dimensional space, defined by a wave-function..$^{20}$ Both the wave-function and the matter density evolve in time: the wave-function by the stochastic GRW dynamics described in Section 3.2, and the matter density by an equation determined by the wave-function, which has therefore the role of governing the behavior of matter. Another spontaneous localization theory with a primitive ontology is called GRWf, and, as in the case of Sf, matter is described directly in space-time: matter is a

\footnotetext{
${ }^{19}$ I am grateful to one of the referees for pressing me on making this point clearer.

${ }^{20}$ This theory was proposed by Ghirardi, Benatti and Grassi (1995).
} 
collection of (discrete) events in space-time, the flashes..$^{21}$ The position of the flashes is determined by the wave-function, which evolves in time according to the modified GRW dynamics: every flash corresponds to one of the spontaneous collapses of the wavefunction, and its space-time location is just the space-time location of that collapse. One can also construct theories, which combine a particle primitive ontology with the GRW dynamics for the wave-function. ${ }^{22}$ One possibility is to consider a theory of particles, which evolve according to the same equation that guides the particles in the pilot-wave theory until a random time; from that time onward, they will still evolve according to the pilot-wave guidance equation but with another wave-function, namely one that has undergone a collapse. In fact, the wave-function evolves according to a stochastic evolution such that at random times it collapses into the actual position of the particle at that time, but 'displaced' at random. ${ }^{23}$ Another possibility for a particle ontology is one in which the particles evolve according to the pilot-wave guidance law between the collapses, like in the theory just discussed. However, at the collapse center, then, all the particles jump at random. (Notice: these theories are hardly to be preferred to the alternatives, given how convoluted they are. However, the point of discussing them it is to explicitly show that it is indeed possible to combine different evolution equations for the wave-function with various kinds of ontology.)

Anyway, how has the situation changed within the primitive ontology approach? Are these theories time-reversal invariant? As we have seen in Section 3.2, Callender had concluded that the spontaneous localization theory was not time-reversal invariant because, being indeterministic, it treats differently the future and the past. If this characterization is correct, then it seems that the situation has not changed much. In fact, while it is true that the GRW evolution equation is not our focus because the wavefunction is not our focus, it still is the case the equation for the mass density in GRWm, say, is indeterministic. Similarly, in the case of GRWf, Sf and Sip, the distribution of the flashes and the configurations of particles are randomly generated by the wave-function (whether it is collapsing or not), thus, it is still seems the case the future and the past are treated differently in the theory.

However, I argue that this conclusion does not necessarily follows: one can still think of these theories as time-reversal invariant. That is, one could reject premise 7 of the argument in Section 3.2. Consider the prototypical example of a stochastic theory, namely the Ehrenfest process (Ehrenfest 1907). It is a discrete-time process which describes the indeterministic dynamics defined by a series of random jumps of the balls between two boxes, one jump at a time. Suppose there are $N$ numbered particles in two

\footnotetext{
${ }^{21}$ The original proposal is due to Bell (1987).

${ }^{22}$ See Allori, Goldstein, Tumulka and Zanghí (2014).

${ }^{23}$ Simpler theories are not be empirically adequate in the sense that they do not (effectively) reproduce the predictions of OQM. See Allori, Goldstein, Tumulka and Zanghí (2014) for more details.
} 
boxes, one on the right and on the left. The state of the system for this example is the list of ball locations in one or the other box at that time. That is, $X=(1, \ldots, N)$ such that the $i$ th entry is 0 if the $i$-th ball is in the right box, and it is 1 if the $i$-th ball is in the left one. Now let us take, randomly, a ball from a box and let us put it in the other one. Thus, the state randomly changes in time. How can one describe the motion of the balls in the Ehrenfest process? A first way to describe it would be to characterize it in pure probabilistic terms: if the system is at a given initial time $t=0$ in the state $X_{0}$, at the time $t$ the system will have certain probability to evolve in one of the $2^{N}$ possible states. Assuming there are only two balls, thus, there are only four possible initial states: $(1,1)$, both balls on the left, $(0,0)$, both balls on the right, $(1,0)$ and $(0,1)$, respectively the first ball on the left and the second on the right and the other way round. If the actual initial state is, say, $(1,1)$, then the system can evolve at time $t=1$ into $(1,0)$ or $(0,1)$ with probability $1 / 2$ each. Assuming the state at $t=1$ is $(1,0)$, then, it can evolve at time $t=2$ into either $(0,0)$ or $(1,1)$, again with $1 / 2$ probability each. And so on.

Using this description, the model seems to lack time-reversal symmetry, for the reasons that we already saw: this description suggests an image of the world in which the past is fixed and the future open. Thus, because of this deep asymmetry, it seems that the notion of time-reversal itself almost ceases to have meaning. This is what Callender presumably has in mind when he discusses the case of indeterministic theories.

Nevertheless, we are not obliged to take this route. ${ }^{24}$ In fact it is possible to reformulate the situation in an equivalent way that allows for the theory to be timereversal invariant. Let us start from the state $X_{0}$ at $t=0$. Consider then the $N$ possible states at $t=1$. At $t=2$, the different accessible states are $N^{2}$, and in general at $t=n$ they are $N^{n}$. In other words, the set of the possible histories of the world that originate from an initial state $X_{0}$ has the shape of a graph. In contrast, in a deterministic dynamics, one state evolves in time into another state, and therefore, the complete history of the world is a single curve in phase space. Instead in an indeterministic theory described in this way, given that multiple states become available to the incoming states, the ways the world can evolve results in a continuous ramification in phase space. Accordingly, the set of all the possible ways the world can be for all the $2^{N}$ possible initial states is the union of the graphs corresponding to the different initial states, which becomes the space of all the possible state of affairs of the world in the case of an indeterministic theory. This branching scenario is a pictorial representation of what having multiple future means.

However, even if there are multiple future available, the system will invariably evolve into only one of them. That is, if we assume a timeless view and look at the space of possible histories, there will always be single histories, namely single sequences of states at different times: one sequence for each path the system at one time will have taken at a later time. Because of this, in this framework, we can define the notion of time-

${ }^{24}$ See also Zanghi (2005). 
reversal in the following way, by defining time-translation first. Let us imagine there is no initial time; a possible way the world can be is a sequence $\left[\ldots, X_{-1}, X_{0}, X_{1}, \ldots\right]$, which is infinite on both the right and the left hand sides. Translating this sequence back in time of, say, a single time unit, means considering another way the world can be, one in which: the state at time $t=1$ is identical with the state at time $t=0$ of the original sequence, namely $X_{0}$; the state at time $t=2$ is identical with the state at time $t=1$ of the original sequence, namely $X_{1}$; and so on. Framed in this way, the time-reversal invariance of the law can be seen using the usual movie metaphor: let $\mathrm{T}$ transform each sequence of states $X=\left[\ldots, X_{1}, X_{2}, X_{3}, \ldots\right]$ in the reversed sequence $T X=\left[\ldots, X_{3}, X_{2}, X_{1}, \ldots\right]$; then if $X$ is a possible state of affairs of the world, then also $T X$ is possible as well. One can see this looking at the graph: consider the yellow sequence in Figure 1: $X=$ $[\ldots,(1,1),(1,0),(0,0), \ldots]$. Then, the corresponding time reversed sequence is: $T X=$ $[\ldots,(0,0),(1,0),(0,0), \ldots]$, which is the one in blue in Figure 1 . This definition of timereversal is the natural generalization of the basic idea that we originally had for deterministic theories based on the movie analogy: the time-reversal of a movie projected forward is the movie projected backwards. In this case, we take a spatio-temporal timeless view: the possible histories of an indeterministic theory are taken in space-time rather than constructed moment after moment in the way proposed by Callender. To summarize, even granting that there are multiple futures open, we are not committed to seeing the world in a ramified, branching picture, as we would by sticking to a moving spotlight picture. Rather, in this timeless view there are always sequences of instantaneous states, and this allows to extend the movie metaphor of time-reversal invariance also in the context of indeterministic theories.

More formally, it seems to me that in the primitive ontology framework, the notion of invariance for indeterministic theories translates in terms of the probability distributions of the primitive ontology because that is what the law for the primitive ontology specifies. Thus, "to say that a theory has a given symmetry is to say that $[\ldots]$ the possible probability distributions on the histories, those that are allowed by the theory, when transformed according to the symmetry, will again be possible probability distributions for the theory" (Allori, Goldstein, Tumulka and Zanghí 2008). In the specific case of GRWm, the history of the primitive ontology, that is the solution of the law for the primitive variables, is the evolution of the matter density field $m$, and a probability distribution on the histories of this primitive ontology is possible if it is the distribution induced on the matter density field by some wave function $\psi$ with probability law given the theory. The case of GRWf and Sf are analogous: a probability distribution for the flashes is possible if induced by the probability law for some wave function $\psi$. The theory is invariant under a given symmetry if the probability distribution on the histories, when transformed according to the symmetry, is the distribution of the transformed histories. In other words, the action of a transformation on every history determines the transformation of a probability distribution on the space of histories. As in the 
deterministic case, the wave function is allowed to change in any way compatible with its relationship to the primitive ontology.

With this definition of time-reversal invariance for indeterministic theories, then, GRWm, GRWf, and Sf are time-reversal invariant. Thus, under the assumptions we made, there is no reason to conclude that time is handed in the quantum world.

\section{Conclusions}

In this paper I have argued that quantum mechanics does not tell us much about the nature of time. I have done so by reviewing the arguments that quantum theories, both in their deterministic and indeterministic form, are not time-reversal invariant (Sections 2 and 3). I have discussed the various reactions to these arguments (Section 4), and I have shown that the argument in the deterministic framework depends on the controversial assumption that the wave-function is a physically real scalar field in configuration space.

Also, I have argued that among the various replies that have been provided to recover the time-reversal invariance of quantum theories, the one that is most satisfactory is to assume that the wave-function does not belong to the state of a physical system and that the wave-function is a ray in Hilbert space, along the line of the so-called primitive ontology approach (Section 5). I have arrived to this second conclusion by showing that the other promising alternative falls short. According to the proponents of this alternative view, quantum theory is invariant because the wave-function is a ray in Hilbert space and the time-reversal operator acts on it according to how its nature prescribes, namely transforming it into its complex conjugate. I have reconstructed this view in my framework as one in which it is denied that the wave-function is a scalar field but in which the wave-function is still part of the ontology of the theory. Then I have argued that it has the counterintuitive and unexplained feature that what one takes to be the ontology of the world depends on whether the state she is reading it from comes from a forward or a backward temporal sequence. In contrast, by removing the wave-function from the state as I propose, this problem disappears (Section 5.1).

Last, I have discussed how the situation changes in indeterministic theories. I have recognized that the problem here is not tightly related to the ontology like in the case above, However, a connection still exists. In fact I show how if one removes the wavefunction for the state as I propose, one can also define the notion of time-reversal invariance for indeterministic theories which may be regarded as a natural extension of the notion for deterministic theories based on the movie analogy, and therefore show how indeterministic quantum theories can be time-reversal invariant after all (Section $5.2)$. 


\section{Acknowledgments}

I am indebted with the participants of the following conferences, who provided me with constructive and helpful feedback on previous versions of this paper: Philosophy of Time Society, Gargnano, BS, Italy (May 11-14, 2014); 'Quantum Time' Conference, Center for Philosophy of Science, Pittsburgh, PA, USA (March 28-29, 2014); The Philosophy of Time Society Session at the APA Pacific Division, San Diego, CA (March 28-March 31, 2018). Similar thanks I owe to the audiences of talks I delivered in the recent past at the University of Macerata and the University at Wisconsin Madison based on ideas expressed in this paper. Finally, I am grateful for the comments provided by four anonymous referees which contributed to sharpen my arguments and to change the structure of the paper definitely for the better. 


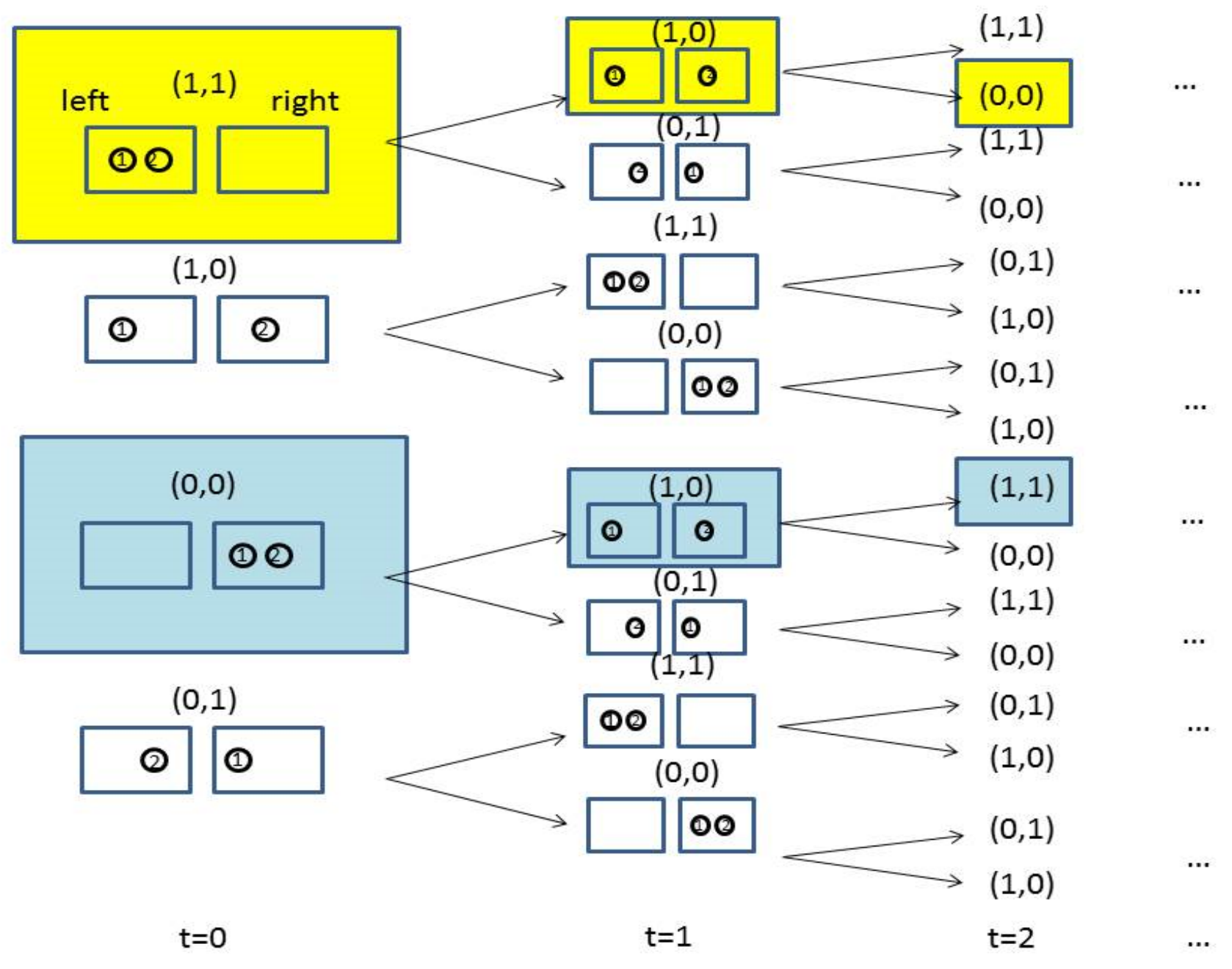

Figure 1: The space of possible histories for the Ehrenfest model with two balls. The states indicated in yellow together constitute one of the possible spatio-temporal histories of the world; the states in blue form the time-reversed history of the yellow states. 


\section{References}

- Aharonov, Yakir and Daniel Rohrlich. 2005. Quantum Paradoxes: Quantum Theory for the Perplexed. Weinheim: Wiley-VCH.

- Aharonov, Yakir, Peter G. Bergmann and Joel L. Lebowitz. 1964. “Time Symmetry in the Quantum Process of Measurement". Physical Review B 134 (6): 1410-1416.

- Albert, David Z. 1996. Elementary Quantum Metaphysics. In: J. Cushing, A. Fine, and S. Goldstein (eds.), Bohmian Mechanics and Quantum Theory: An Appraisal: 277-284. Kluwer.

- Albert, David Z. 2000. Time and Chance. Harvard University Press.

- Albert, David Z. 2013. “Wave Function Realism”. In: D.Z. Albert, and A. Ney (eds.) The Wave Function: Essays in the Metaphysics of Quantum Mechanics: 52-57. New York: Oxford University Press.

- Albert, David Z. 2015. After Physics. Harvard University Press.

- Albert, David Z. and Alissa Ney (eds.). 2013 The Wave Function: Essays on the Metaphysics of Quantum Mechanics. Oxford University Press.

- Allori, Valia. 2018. “A New Argument for the Nomological Interpretation of the Wave Function: The Galilean Group and the Classical Limit of Nonrelativistic Quantum Mechanics." International Studies in the Philosophy of Science 31 (2): 177-188.

- Allori, Valia. 2015a. "Primitive Ontology in a Nutshell." International Journal of Quantum Foundations 1 (3): 107-122 (2015).

- Allori, Valia. 2015b. "Maxwell's Paradox: Classical Electrodynamics and its Time Reversal Invariance". Analytica 1:1-19

- Allori, Valia. 2013a: "Primitive Ontology and the Structure of Fundamental Physical Theories". In: D.Z. Albert, A. Ney (eds.), The Wave Function: Essays in the Metaphysics of Quantum Mechanics: 58-75. Oxford University Press.

- Allori, Valia. 2013b: "On the Metaphysics of Quantum Mechanics". In: S. Lebihan (ed.), Precis de la Philosophie de la Physique: 116-151. Paris: Vuibert.

- Allori, Valia, Sheldon Goldstein, Roderich Tumulka and Nino Zanghì. 2008. "On the Common Structure of Bohmian Mechanics and the Ghirardi-Rimini-Weber Theory". British Journal for the Philosophy of Science 59: 353-389.

- Allori, Valia, Sheldon Goldstein, Roderich Tumulka and Nino Zanghì. 2011. "ManyWorlds and Schrödinger's First Quantum Theory". The British Journal for the Philosophy of Science 62 (1): 1--27.

- Allori, Valia, Sheldon Goldstein, Roderich Tumulka and Nino Zanghì. 2014. "Predictions and Primitive Ontology in Quantum Foundations: A Study of Examples". The British Journal for the Philosophy of Science 65 (2): 323-352.

- Arntzenius, Frank. 2004. "Time-reversal Operation, Representation of the Lorentz Group and the Direction of Time." Studies in Histories and Philosophy of Modern Physics 35(1): 31-43. 
- Bell, John S. 1987. Speakable and Unspeakable in Quantum Mechanics. Cambridge: Cambridge University Press.

- Bhogal, Harjit and Zee R. Perry. 2017. "What the Humean should say about Entanglement". Nous 51(1), 74-94.

- Bohm, David. 1952. "A Suggested Interpretation of the Quantum Theory in Terms of "Hidden" Variables", I and II. Physical Review 85: 166-193.

- Brading, Katherine and Elena Castellani. 2003. Symmetries in Physics: Philosophical Reflections. Cambridge: Cambridge University Press.

- Bricmont, Jean. 2017. Making Sense of Quantum Mechanics. Springer.

- Callender, Craig. 2000. "Is Time 'Handed' in the Quantum World?" Proceedings of the Aristotelian Society: 247-269.

- Callender, Craig. 2015. “One World, One Beable”. Synthese, 192(10): 3153-3177.

- de Broglie, Louis. 1928. "La Nouvelle Dynamique des Quanta". In: J.Bordet (ed.) Electrons et Photons: Rapports et Discussions du Cinquième Conseil de Physique. GauthierVillars: 105-132.

- Deutsch, David. 1996. The Fabric of Reality. New York: The Penguin Press.

- De Witt, Bryce S. 1970. "Quantum Mechanics and Reality." Physics Today 23(9): 3040.

- Dürr, Detlef, Sheldon Goldstein and Nino Zanghi.1992. "Quantum Equilibrium and the Origin of Absolute Uncertainty". Journal of Statistical Physics 67: 843-907.

- Dürr, Detlef, Sheldon Goldstein, and Nino Zanghì. 1997. "Bohmian Mechanics and the Meaning of the Wave Function". In R.S. Cohen, M. Horne, and J. Stachel (eds.) Experimental Metaphysics - Quantum Mechanical Studies for Abner Shimony, Volume One; Boston Studies in the Philosophy of Science 193. Kluwer Academic Publishers.

- Earman, John. 2002. "What Time-reversal Invariance is and Why it Matters." International Studies in the Philosophy of Science 16: 245-264.

- Ehrenfest, Paul and Tatjana Ehrenfest. 1907. "Über zwei bekannte Einwände gegen das Boltzmannsche H-Theorem". Physikalishce Zeitschrift 8: 311-314.

- Esfeld, Michael, Dustin Lazarovici, Mario Hubert, Detlef Dürr. 2014. "The Ontology of Bohmian Mechanics". The British Journal for the Philosophy of Science 65(4): 773-796.

- Everett, High. 1957. "Relative State Formulation of Quantum Mechanics". Review of Modern Physics 29: 454-462.

- Ghirardi, GianCarlo, Alberto Rimini, Tulio Weber, 1986. “Unified Dynamics for Microscopic and Macroscopic Systems". Physical Review D 34: 470-491.

- Ghirardi, GianCarlo, Fabio Benatti and Renata Grassi.1995. "Describing the Macroscopic World: Closing the Circle within the Dynamical Reduction Program". Foundations of Physics 25: 5-38.

- Goldstein, Sheldon and Stefan Teufel. 2001. “Quantum Spacetime without Observers: Ontological Clarity and the Conceptual Foundations of Quantum Gravity." In. C. 
Callender, and N. Huggett (eds.), Physics meets Philosophy at the Planck Scale. Cambridge University Press.

- Goldstein, Sheldon, and Nino Zanghí. 2013. "Reality and the Role of the Wave Function in Quantum Theory". In: D.Z. Albert and A. Ney (eds.), The Wave Function: Essays on the Metaphysics of Quantum Mechanics: 91-109. Oxford University Press.

- Hossenfelder, Sabine. 2018. Lost in Math: How Beauty Leads Physics Astray. Basic Books.

- Lewis, Peter J. 2004. "Life in Configuration Space." British Journal for the Philosophy of Science 55: 713-729.

- Lewis, Peter J. 2013."Dimensions as illusions." In: D.Z. Albert, and A. Ney (eds.), The Wave Function: Essays in the Metaphysics of Quantum Mechanics: 110-125. New York: Oxford University Press.

- Lewis, Peter J. 2017. "On the Status of Primitive Ontology." In S. Gao (ed.), Collapse of the Wave Function Models, Ontology, Origin, and Implications: 154-166. Cambridge University Press.

- Lockwood, Michael, 1989. Mind, Brain and the Quantum. Oxford: Basil Blackwell.

- Malament, David. 2004. “On the Time-reversal Invariance of Classical Electromagnetic Theory." Studies in Histories and Philosophy of Modern Physics B 35: 295315.

- Miller, Elizabeth. 2014: “Quantum Entanglement, Bohmian Mechanics, and Humean Supervenience". Australasian Journal of Philosophy 92(3): 567-83.

- Monton, Bradley. 2002. "Wave Function Ontology". Synthese 130, 265-277.

- von Neumann, John. 1932: Mathematische Grundlagen der Quantenmechanik. Springer, Berlin (1932). English translation: Mathematical Foundations of Quantum Mechanics, Princeton University Press (1955).

- Monton, Bradley 2013. "Against 3-N-Dimensional Space." In: D. Z. Albert and A. Ney (eds.). The Wave Function: Essays in the Metaphysics of Quantum Mechanics: 154167. New York: Oxford University Press.

- Ney, Alyssa. 2012. "The Status of Our Ordinary Three Dimensions in a Quantum Universe". Nous 46 (3): 525-560.

- Ney, Alyssa. 2013. "Ontological Reduction and the Wave Function Ontology". In: D.Z. Albert, and A. Ney (eds.), The Wave Function: Essays in the Metaphysics of Quantum Mechanics: 168-183. Oxford University Press.

- North, Jill. 2008. "Two Views of Time-reversal”. Philosophy of Science 75 (2): 201-223.

- North, Jill. 2013. "The Structure of the Quantum World." In:

- D.Z. Albert, and A. Ney (eds.), The Wave Function: Essays in the Metaphysics of Quantum Mechanics: 184-202. New York: Oxford University Press.

- Pearl, Philip. 1989. "Combining Stochastic Dynamical State-vector Reduction with Spontaneous Localization". Physical Review A 39 (5): 2277-2284.

- Popper, Karl 1967. "Quantum Theory without 'the Observer'". In: M. Bunge (ed.) Quantum Theory and Reality: 1-12. New York, Springer. 
- Roberts, Bryan W. 2017. Three Myths about Time Reversal in Quantum Theory. Philosophy of Science 84 (2): 315-334.

- Saunders, Simon. 1993. "Decoherence, Relative States, and Evolutionary Adaptation". Foundations of Physics 23: 1553-1585.

- Skow, Braford. 2010. "On a Symmetry Argument for the Guidance Equation in Bohmian Mechanics." International Studies in the Philosophy of Science 24 (4):393-410.

- Suàrez, Mauricio. 2015. "Bohmian Dispositions". Synthese 192(10): 3203-28.

- Valentini, Antony. 1991. "Signal-locality, Uncertainty, and the Sub-quantum Htheorem." Physics Letters A 156(5): 5-11.

- Wallace, David. 2012. The Emergent Multiverse. Oxford: University Press.

- Wigner, Eugene. 1959. Group Theory and Its Application to the Quantum Mechanics of Atomic Spectra. NY: Academic Press.

- Zanghì, Nino. 2005. "I Fondamenti Concettuali dell'Approccio Statistico in Fisica". In: Valia Allori, Mauro Dorato, Federico Laudisa, and Nino Zanghì (eds.): La Natura Delle Cose. Introduzione ai Fundamenti e alla Filosofia della Fisica: 139-228. Roma: Carocci editore. 